Pacific Journal of Mathematic 


\title{
FIXED POINT PROPERTIES AND INVERSE LIMIT SPACES
}

\author{
SHWU-Yeng T. LIN
}

The purpose of this paper is to prove that if $\left(X_{\lambda}, \pi_{\lambda \mu}, \Lambda\right)$ is an inverse system of compact Hausdorff spaces such that each $X_{\lambda}$ has the fixed point property for the continuous multivalued functions and each projection map is surjective, then the inverse limit space also has the fixed point property for the continuous multi-valued functions.

A topological space $X$ is said to have the f.p.p. (fixed point property) if for every continuous (single-valued) function $f: X \rightarrow X$ there exists some $x$ in $X$ such that $x=f(x)$. Hamilton [3] has proved that the chainable metric continua have the f.p.p. A topological space $X$ is said to have the F.p.p. (fixed point property for multi-valued functions) if every continuous (see Definition 1) multi-valued function $F: X \rightarrow X$ has a fixed point; that is, there exists some point $x$ in $X$ such that $x \in F(x)$. If a space has the F.p.p. then it has the f.p.p,, but the converse need not be true [12]. Mardesic [8] has exhibited an inverse sequence, $\left(X_{m}, \pi_{m n}\right)$, of polyhedra, $X_{m}$, such that all $X_{m}$ have the f.p.p. and all bonding maps $\pi_{m n}$ are surjective, but the inverse limit space, $\lim \left(X_{m}, \pi_{m n}\right)$, fails to have the f.p.p. This answered an open question raised by Mioduszewski and Rochowski [9 and 10], in the negative. Thus, our result stated in the first paragraph serves as an interesting counter-theorem to the result of Mardesic [op. cit.]. As a corollary, we obtain Ward's generalization [13] of the Hamilton theorem [op. cit.] that every metric chainable continuum has the F.p.p. In effect, our result is stronger than that of ward, since it includes some of the nonmetrizable chainable continua as well.

1. Preliminaries. In all that follows, all spaces are assumed to be Hausdorff spaces. A multifunction, $F: X \rightarrow Y$, from a space $X$ to a space $Y$ is a point-to-set correspondence such that, for each $x \in X$, $F(x)$ is a subset of $Y$. For any $y \in Y$, we write $F^{-1}(y)$ for the set $\{x \in X \mid y \in F(x)\}$. If $A \subset X$ and $B \subset Y$, then $F(A)=\cup\{F(x) \mid x \in A\}$ and $F^{-1}(B)=\cup\left\{F^{-1}(y) \mid y \in B\right\}$.

Definition 1. A multifunction, $F: X \rightarrow Y$, is said to be continuous if and only if (i) $F(x)$ is closed for each $x$ in $X$, (ii) $F^{-1}(B)$ is closed for each closed set $B$ in $Y$, and (iii) $F^{-1}(V)$ is open for each open set $V$ in $Y$.

Our definition of continuity here is weaker than that of Berge [1, 
p. 109], but these two definitions coincide when the range space $Y$ is compact.

A proof of the following lemma may be found in Berge [1, Th. 3, p. 110].

Lemma 1. If $f: X \rightarrow Y$ is a continuous multifunction and if $A$ is a compact subset of $X$ such that $F(a)$ is compact for each $a \in A$, then $F(A)$ is compact.

Definition 2. The triple, $\left(X_{\lambda}, \pi_{\lambda \mu}, A\right)$, is an inverse system of spaces if and only if:

(i) $A$ is a directed set directed by $<$,

(ii) for each $\lambda \in \Lambda, X_{\lambda}$ is a (Hausdorff) space,

(iii) if $\lambda>\mu, \pi_{\lambda \mu}$ is a continuous function of $X_{\lambda}$ to $X_{\mu}$,

(iv) if $\lambda>\mu$ and $\mu>\nu$, then $\pi_{\lambda \nu}=\pi_{\mu \nu} \pi_{\lambda \mu}$.

Each function $\pi_{\lambda \mu}$ is called a bonding map. If $\lambda$ is in $\Lambda$, let $S_{\lambda}$ be the subset of the Cartesian product $P\left\{X_{\lambda} \mid \lambda \in A\right\}$ defined by

$$
S_{\lambda}=\left\{x \mid \text { if } \lambda>\mu \text { then } \pi_{i \mu} x(\lambda)=x(\mu)\right\},
$$

where $x(\lambda)$ denotes the $\lambda$-th coordinate of $x$.

Definition 3. The inverse limit space, $X_{\infty}$, of the inverse system of spaces $\left(X_{\lambda}, \pi_{\lambda \mu}, \Lambda\right)$ is defined to be

$$
\bigcap\left\{S_{\lambda} \mid \lambda \in \Lambda\right\}
$$

endowed with the relative topology inherited from the product topology for $P\left\{X_{\lambda} \mid \lambda \in \Lambda\right\}$. In notation, we shall write $X_{\infty}$ and $\lim \left(X_{\lambda}, \pi_{\lambda, !}, \Lambda\right)$ interchangeably for the inverse limit space defined above.

We write $p_{\lambda}: P\left\{X_{\lambda} \mid \lambda \in \Lambda\right\} \rightarrow X_{\lambda}$ for the $\lambda$-th projection of $P\left\{X_{\lambda} \mid \lambda \in \Lambda\right\}$, i.e., $p_{\lambda}(x)=x(\lambda)$ for all $x$ in $P\left\{X_{\lambda} \mid \lambda \in \Lambda\right\}$; the restriction $p_{\lambda} \mid X_{\infty}$ will be denoted by $\pi_{2}$, which will be called a projection map. It is readily seen from the definition that an element $x$ of $P\left\{X_{\lambda} \mid \lambda \in \Lambda\right\}$ is in $X_{\infty}$ if and only if $\pi_{\lambda \mu} p_{\lambda}(x)=p_{\mu^{\prime}}(x)$ whenever $\lambda>\mu$. A more detailed account of inverse limit spaces may be found in Lefschetz [6], Capel [2] and Mardešić [7].

The following known results (see, e.g., [2], [6]) will be used.

Lemma 2. (i) The collection $\left\{\pi_{i}^{-1}\left(U_{i}\right) \mid \lambda \in \Lambda\right.$ and $U_{i}$ is an open subset of $X_{\lambda}$ forms a basis for the topology of $X_{\infty}$.

(ii) The inverse limit space, $X_{\infty}$, is Hausdorff; if $\lambda \in \Lambda, S_{i}$ is a closed subset of $P\left\{X_{\lambda} \mid \lambda \in \Lambda\right\}$ so that $X_{\infty}$ is closed in $P\left\{X_{\lambda} \mid \lambda \in \Lambda\right\}$.

(iii) If $X_{\lambda}$ is compact for each $\lambda$ in $\Lambda$, then $X_{\infty}$ is compact; if, in addition, each $X_{\lambda}$ is nonvoid, then $X_{\infty}$ is nonvoid.

(iv) If $X_{\lambda}$ is a continuum for each $\lambda \in \Lambda$, then the inverse limit 
space is a continuum.

Lemma 3. If $A$ is a compact subset of $X_{\infty}$ and if $\pi_{\lambda \mu}^{\prime}=\pi_{\lambda_{\mu}} \mid \pi_{\lambda}(A)$, then $\left(\pi_{\lambda}(A), \pi_{\lambda \mu}^{\prime}, A\right)$ is an inverse system of spaces such that $A=$ $\lim \left(\pi_{\lambda}(A), \pi_{\lambda \mu}^{\prime}, \Lambda\right)$, and each bonding map $\pi_{\lambda_{\mu}}^{\prime}$ is surjective.

2. Main results. In the sequel, since we are only interested in compact spaces, each projection map $\pi_{2}$ will be assumed to be surjective; for if otherwise, by virtue of Lemma 3, each $X_{\lambda}$ may be replaced by $\pi_{\lambda}\left(X_{\infty}\right)$ without disturbing the resulting inverse limit space. We are now ready to state our main result.

MaIN Theorem. Let $\left(X_{\lambda}, \pi_{\lambda, \mu}, A\right)$ be an inverse system of compact spaces such that each $X_{\lambda}$ has the F.p.p., then the inverse limit space $X_{\infty}$ also has the F.p.p.

We divide the proof of this theorem into the following steps. In Lemmas 4,5 and $6, X_{\infty}$ will be the inverse limit space of the inverse system $\left(X_{\lambda}, \pi_{\lambda \mu}, \Lambda\right)$ of compact spaces.

Lemma 4. If $F: X_{\infty} \rightarrow X_{\infty}$ is a continuous multifunction, define $F_{\lambda}: X_{\lambda} \rightarrow X_{\lambda}$ by $F_{\lambda}=\pi_{\lambda} F \pi_{\lambda}^{-1}$ for each $\lambda$, then $F_{\lambda}$ is a continuous multifunction.

Proof. (i ) By Lemma $1, F\left(\pi^{-1}(t)\right)$ is compact in $X_{2}$ for each $t$ in $X_{\lambda}$, and consequently each $F_{\lambda}(t)$ is closed in $X_{\lambda}$.

(ii) If $C_{\lambda}$ is a closed subset of $X_{\lambda}$, then $F_{\lambda}^{-1}\left(C_{\lambda}\right)$ is closed. For, the set $F^{-1} \pi_{\lambda}^{-1}\left(C_{\lambda}\right)$ is closed in $X_{\infty}$ and hence compact; therefore $\pi_{\lambda} F^{-1} \pi_{\lambda}^{-1}\left(C_{\lambda}\right)=F_{\lambda}^{-1}\left(C_{\lambda}\right)$ is compact and hence closed.

(iii) Since each $\pi_{\lambda}$ is also an open map, as a dual of (ii) above, $F_{\lambda}^{-1}\left(U_{\lambda}\right)$ is open for each open set $U_{\lambda}$ in $X_{\lambda}$.

Thus, by (i), (ii) and (iii) above, $F_{\lambda}: X_{\lambda} \rightarrow X_{\lambda}$ is continous.

LEMMA 5. $F: X_{\infty} \rightarrow X_{\infty}$ be a continuous multifunction, let $F_{\lambda}: X_{\lambda} \rightarrow X_{\lambda}$ be defined as in Lemma 4. Then, for each $x$ in $X_{\infty}$,

(i ) $\left(F_{\lambda} \pi_{\lambda}(x), \pi_{\lambda \mu}, A\right)^{1}$ and $\left(\pi_{\lambda} F(x), \pi_{\lambda \mu}, \Lambda\right)$ are inverse systems of compact spaces,

(ii) $\lim \left(F_{\lambda} \pi_{\lambda}(x), \pi_{\lambda_{\ell !}}, \Lambda\right)=\lim \left(\pi_{\lambda} F(x), \pi_{\lambda \mu}, \Lambda\right)$,

(iii) $\overleftarrow{F(x)}=\longleftarrow \lim _{\longleftarrow}\left(F_{\lambda} \pi_{\lambda}(x), \pi_{\lambda, \mu}, \Lambda\right)$

Proof. (i) It is obvious that each $F_{i} \pi_{\lambda}(x)$ is compact. To show that $\left(F_{\lambda} \pi_{\dot{\lambda}}(x), \pi_{\lambda \mu}, A\right)$ forms an inverse system, it suffices to show $\pi_{\lambda \mu} F_{\lambda} \pi_{\lambda}(x) \subset F_{\mu} \pi_{\mu}(x)$ whenever $\lambda>\mu$. To this end we first observe

${ }^{1}$ For simplicity in symbolism, henceforth if $A \subset \lim \left(X_{\lambda}, \pi_{\lambda \mu}, \Lambda\right)$, then $\left(\pi_{i}(A), \pi_{\lambda \mu}, A\right)$ will mean $\left(\pi_{\lambda}(A), \pi_{\lambda \mu} \mid \pi_{\lambda}(A), A\right)$. 


$$
\pi_{\lambda}(x) \in\left(\pi_{\lambda \mu}^{-1} \pi_{\lambda \mu}\right) \pi_{\lambda}(x)=\pi_{\lambda \mu}^{-1} \pi_{\mu}(x),
$$

since $\pi_{\lambda \mu} \pi_{\lambda}=\pi_{\mu}$. From this, with some computations,

$$
\pi_{\lambda \mu} F_{i j} \pi_{\lambda}(x) \subset F_{\mu} \pi_{\mu}(x)
$$

follows.

The fact that $\left(\pi_{2} F(x), \pi_{\lambda_{\mu}}, \Lambda\right)$ forms an inverse system follows from Lemma 3.

(ii) For each $\lambda \in \Lambda$ and any $x \in X_{\infty}$, we have

$$
\pi_{\lambda} F(x) \subset \pi_{\lambda} F \pi_{\lambda}^{-1} \pi_{\lambda}(x)=\left(\pi_{\lambda} F \pi_{\lambda}^{-1}\right) \pi_{\lambda}(x)=F_{\lambda_{\lambda}} \pi_{\lambda}(x),
$$

and thus,

$$
\lim _{\longleftarrow}\left(\pi_{\lambda} F(x), \pi_{\lambda \mu}, \Lambda\right) \subset \lim _{\longleftarrow}\left(F_{i} \pi_{\lambda}(x), \pi_{\lambda \mu}, \Lambda\right) .
$$

To prove the other inclusion, we show

$$
X_{\infty}-\underset{\lim }{\longleftarrow}\left(\pi_{\lambda} F(x), \pi_{\lambda \mu}, \Lambda\right) \subset X_{\infty}-\lim _{\longleftarrow}\left(F_{\lambda} \pi_{\lambda}(x), \pi_{\lambda \mu}, \Lambda\right) .
$$

Let $y$ be in $X_{\infty}-\lim \left(\pi_{\lambda} F(x), \pi_{\lambda, \mu}, A\right)$, then by Lemma 3 there exists a $\mu \in \Lambda$ such that $\pi_{\mu} \overleftarrow{(y)} \notin \pi_{\mu} F(x)$. Let $U_{\mu}$ and $V_{\mu}$ be two disjoint open sets in $X_{\mu}$ such that

$$
\pi_{\mu}(y) \in U_{\mu} \text { and } \pi_{\mu} F(x) \subset V_{\mu}
$$

so that

$$
F(x) \subset \pi_{\mu}^{-1}\left(V_{\mu}\right) \text {. }
$$

If follows then from Lemma 2(i) and the continuity of $F$ that there exists a $\delta \in \Lambda$ and an open set $U_{\delta}$ in $X_{\delta}$ such that $x \in \pi_{\delta}^{-1}\left(U_{\delta}\right)$, and

$$
F\left(\pi_{\hat{o}}^{-1}\left(U_{\delta}\right)\right) \subset \pi_{\mu}^{-1}\left(V_{\mu}\right) .
$$

Since $\Lambda$ is directed, there is a $\lambda_{0} \in \Lambda$ such that $\lambda_{0}>\mu$ and $\lambda_{0}>\delta$, we shall use this $\lambda_{0}$ throughout the proof of lemma. If we denote $U_{\lambda_{0}}=$ $\pi_{\lambda_{0} \delta}^{-1}\left(U_{\delta}\right)$ and using the equality $\pi_{\delta}^{-1}=\pi_{\hat{i}_{0}}^{-1} \pi_{\lambda_{0} \delta}^{-1}$, then $\left({ }^{*}\right)$ may be rewritten as

$$
F\left(\pi_{\lambda_{0}}^{-1}\left(U_{\lambda_{0}}\right)\right) \subset \pi_{\mu}^{-1}\left(V_{\mu}\right),
$$

and hence

$$
F_{\lambda_{0}}\left(U_{\lambda_{0}}\right)=\pi_{\lambda_{0}} F \pi_{\lambda_{0}}^{-1}\left(U_{\lambda_{0}}\right) \subset \pi_{\lambda_{0}} \pi_{\mu}^{-1}\left(V_{\mu}\right)=\pi_{\lambda_{0}}\left(\pi_{\lambda_{0} \mu} \pi_{\lambda_{2}}\right)^{-1}\left(V_{\mu}\right)=\pi_{\lambda_{0} \mu^{\mu}}^{-1}\left(V_{\mu}\right) .
$$

In particular,

$$
F_{\lambda_{0}} \pi_{\lambda_{0}}(x) \subset \pi_{\lambda_{0} \mu^{\prime \prime}}^{-1}\left(V_{\mu}\right) .
$$

Similarly, one obtains $\pi_{\lambda_{0}}(y) \in \pi_{\lambda_{0^{\mu}}}^{-1}\left(U_{\mu}\right)$.

Since $\pi_{\lambda_{0} \mu^{\mu}}^{-1}\left(V_{\mu}\right)$ and $\pi_{\lambda_{0} \mu^{\mu}}^{-1}\left(U_{\mu}\right)$ are disjoint, $\pi_{\lambda_{0}}(y) \in F_{\lambda_{0} 0} \pi_{\lambda_{0}}(x)$. From this we 
conclude $y \notin \lim \left(F_{i} \pi_{\lambda}(x), \pi_{\lambda \mu}, \Lambda\right)$, as desired.

(iii) This follows immediately from (ii) above and Lemma 3.

LEMMA 6. Let $F: X_{\infty} \rightarrow X_{\infty}$ be a continuous multifunction, let $F_{\lambda}: X_{\lambda} \rightarrow X_{\lambda}$ be defined as in Lemma 4. Let $E_{\lambda}=\left\{e_{\lambda} \mid e_{\lambda} \in X_{\lambda}\right.$ and $\left.e_{\lambda} \in F_{\lambda}\left(e_{\lambda}\right)\right\}$ then $\left(E_{\lambda}, \pi_{\lambda_{\mu}}, \Lambda\right)$ forms an inverse system.

Proof. It suffices to prove $\pi_{\lambda \mu}\left(E_{\lambda}\right) \subset E_{\mu}$ whenever $\lambda>\mu$, which follows in a routine way.

Proof of main theorem. Since each $X_{\lambda}$ has the F.p.p. and, by Lemma 4, each $F_{\lambda}: X_{\lambda} \rightarrow X_{\lambda}$ is continuous, each $E_{\lambda}$ is closed and nonvoid. By Lemma $6,\left(E_{\lambda}, \pi_{\lambda \mu}, \Lambda\right)$ is an inverse system of compact spaces, so it has a nonvoid inverse limit space $\lim \left(E_{\lambda}, \pi_{\lambda \mu}, \Lambda\right)$. We now conclude the proof by showing that each $x$ in $\overleftarrow{\lim }\left(E_{\lambda}, \pi_{\lambda \mu}, \Lambda\right)$ is a fixed point under $F$; i.e., $x \in F(x)$. If $x$ is in $\left.\lim \overleftarrow{(E}_{\lambda}, \pi_{\lambda \mu}, \Lambda\right)$, then $\pi_{\lambda}(x) \in E_{\lambda}$ for all $\lambda \in \Lambda$; i.e., $\pi_{\lambda}(x) \in F_{\lambda} \pi_{\lambda}(x)$ for all $\overleftarrow{\lambda \in \Lambda}$. Consequently, by Lemmas 3 and 5 , we have

$$
x=\lim _{\longleftarrow}\left(\pi_{\lambda}(x), \pi_{\lambda \mu}, \Lambda\right) \in \lim _{\longleftarrow}\left(F_{\lambda} \pi_{\lambda}(x), \pi_{\lambda \mu}, \Lambda\right)=F(x) .
$$

Since the main theorem fails for single-valued functions, it should be pointed out that why the above argument breaks down in the singlevalued case: given any continuous multifunction $F: X_{\infty} \rightarrow X_{\infty}$, each induced $F_{\lambda}$ is again a continuous multifunction and hence has a fixed point; this is crucial to the proof. In the single-valued case, however, it does not follow in general that $F_{\lambda}$ is single-valued and hence $F_{\lambda}$ may not have a fixed point.

In fact, with the assumption of the main theorem and the notation of Lemma 6 together with the notation $E=\{x \mid x \in F(x)\}$, we can make the following sharper assertion.

Theorem. $\quad E=\lim \left(E_{\lambda}, \pi_{\lambda \mu}, \Lambda\right)$.

Proof. From the proof of the main Theorem, we have $E \supset \lim \left(E_{\lambda}\right.$, $\left.\pi_{\lambda \mu}, A\right)$. It remains to be proved that

$$
E \subset \lim _{\longleftarrow}\left(E_{\lambda}, \pi_{\lambda \mu}, \Lambda\right) .
$$

Let $x$ be in $E$, then $x \in F(x)$ and therefore, for all $\lambda \in \Lambda$,

$$
\pi_{\lambda}(x) \in \pi_{\lambda} F(x) \subset \pi_{\lambda} F\left(\pi_{\lambda}^{-1} \pi_{\lambda}\right)(x)=F_{\lambda}\left(\pi_{\lambda}(x)\right) .
$$

That is, $\pi_{\lambda}(x) \in E_{\lambda}$ for all $\lambda$; consequently, by Lemma $3, E \subset \lim \left(E_{\lambda}, \pi_{\lambda \mu}, \Lambda\right)$. A chain $\left(U_{1}, U_{2}, \cdots, U_{n}\right)$ is a finite sequence of sets $\overleftarrow{U}_{i}$ such that 
$U_{i} \cap U_{j} \neq \square$ if and only if $|i-j| \leqq 1$, where $\square$ denotes the empty set. A Hausdorff space $X$ is said to be chainable if to each open cover $\mathscr{Y}$ of $X$ there is a finite open cover $\mathscr{U}=\left(U_{1}, U_{2}, \cdots, U_{n}\right)$ such that (i) $\mathscr{U}$ refines $\mathscr{V}$; (ii) $\mathscr{U}=\left(U_{1}, U_{2}, \cdots, U_{n}\right)$ forms a chain. It follows that a chainable space is a continuum. It is implicit in the paper of Isbell [5] that each metrizable chainable continuum is the inverse limit space of a sequence of (real) arcs. This together with a theorem of Strother [12] that a bounded closed interval of the real numbers has the F.p.p. implies the following result of Ward [13] as a consequence of our main theorem.

Corollary [13]. Each chainable metric continuum has the F.p.p.

Examples of inverse limit spaces of inverse systems of real arcs exist which are not metrizable; for instance, the long line [4, p. 55] is one such.

We are indebted to the paper of Professor Rosen [11], and to Professor A. D. Wallace for his kind encouragement.

\section{BIBLIOGRAPHY}

1. Claude Berge Topological Spaces, Macmillan Company, New York, 1963.

2. C. E. Capel, Inverse limit spaces, Duke Math. J. 21 (1954), 233-245.

3. O. H. Hamilton, A fixed point theorem for pseudo-arcs and certain other metric continua, Proc. Amer. Math. Soc. 2 (1951), 173-174.

4. J. G. Hocking and Gail S. Young, Jr., Topology, Addison-Wesley Publishing Company, Reading, Mass., 1961.

5. J. R. Isbell, Embeddings of inverse limits, Ann. of Math. 70 (1959), 73-84.

6. S. Lefschetz, Algebraic Topology, Amer. Math. Soc. Colloq. Publ., no. 27, New York, 1942.

7. S. Mardešić, On inverse limits of compact spaces, Glasnik Mat. Fiz. Astr. 13 (1958), 249-255.

8. - Mappings of inverse systems, Glasnik Mat. Fiz. Astr. 18 (1963), 241-254.

9. J. Mioduszewski and M. Rochowski, Remarks on fixed point theorem for inverse limit spaces, Colloq. Math. 10 (1962), 67-71.

10. - Remarks on fixed point theorem for inverse limit spaces, Proc. Sympos. General Topology and Its Relations to Modern Analysis and Algebra, Prague, 1962, 275-276.

11. R. H. Rosen, Fixed points for multi-valued functions on snake-like continua, Proc. Amer. Math. Soc., 10 (1959), 167-173.

12. W. L. Strother, On an open question concerning fixed points, Proc. Amer. Math. Soc. 4 (1953), 988-993.

13. L. E. Ward, Jr., A fixed point theorem, Amer. Math. Monthly 65 (1958), 171-172.

Received June 29, 1967.

UNIVERSity OF SOUTh Florida

TAMPA, FLORIDA 


\section{PACIFIC JOURNAL OF MATHEMATICS}

\section{EDITORS}

H. ROYDEN

Stanford University

Stanford, California

\section{J. P. Jans}

University of Washington

Seattle, Washington 98105

\section{J. DUGUNDJI}

Department of Mathematics University of Southern California Los Angeles, California 90007

\section{RICHARD ARENS}

University of California

Los Angeles, California 90024

\section{ASSOCIATE EDITORS}

E. F. BECKENBACH

B. H. NEUManN

F. WOLF

K. YosIDA

\section{SUPPORTING INSTITUTIONS}

UNIVERSITY OF BRITISH COLUMBIA CALIFORNIA INSTITUTE OF TECHNOLOGY UNIVERSITY OF CALIFORNIA MONTANA STATE UNIVERSITY UNIVERSITY OF NEVADA NEW MEXICO STATE UNIVERSITY OREGON STATE UNIVERSITY UNIVERSITY OF OREGON OSAKA UNIVERSITY UNIVERSITY OF SOUTHERN CALIFORNIA
STANFORD UNIVERSITY

UNIVERSITY OF TOKYO UNIVERSITY OF UTAH WASHINGTON STATE UNIVERSITY UNIVERSITY OF WASHINGTON AMERICAN MATHEMATICAL SOCIETY CHEVRON RESEARCH CORPORATION TRW SYSTEMS NAVAL WEAPONS CENTER 


\section{Pacific Journal of Mathematics}

\section{Vol. 25, No. $1 \quad$ September, 1968}

Glen Eugene Bredon, Cosheaves and homology................... 1

Robin Ward Chaney, A chain rule for the transformation of integrals in

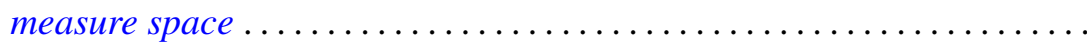

Colin W. Clark, On relatively bounded perturbations of ordinary differential operators................................... 59

John Edwin Diem, A radical for lattice-ordered rings.............. 71

Zeev Ditzian, On a class of convolution transforms ................ 83

Dennis Garoutte and Paul Adrian Nickel, A note on extremal properties characterizing weakly $\lambda$-valent principal functions............. 109

Shwu-Yeng Tzeng Lin, Fixed point properties and inverse limit spaces . . . 117

John S. Lowndes, Some dual series equations involving Laguerre polynomials ................................. 123

Kirti K. Oberai, Sum and product of commuting spectral operators ....... 129

J. N. Pandey and Armen H. Zemanian, Complex inversion for the generalized convolution transformation..................... 147

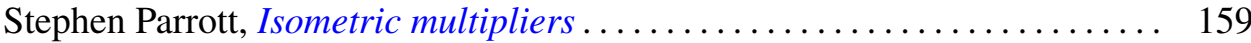

Manoranjan Prasad, Note on an extreme form .................. 167

Maciej Skwarczyński, A representation of a bounded function as infinite product in a domain with Bergman-Shilov boundary surface ......... 177

John C. Taylor, The Šilov boundary for a lattice-ordered semigroup ...... 185 Donald Reginald Traylor and James Newton Younglove, On normality and pointwise paracompactness ............................. 193

L. Tzafriri, Quasi-similarity for spectral operators on Banach spaces ... 